\title{
Radio environment map-based cognitive Doppler spread compensation algorithms for high-speed rail broadband mobile communications
}

\author{
Jinxing Li and Youping Zhao*
}

\begin{abstract}
Recently, there is an increasing yet challenging demand on broadband mobile communications for high-speed trains. In this article, cognitive Doppler spread compensation algorithms are proposed for high-speed rail broadband mobile communications, which make use of the dedicated radio environment map (REM) for railway to compensate the time-varying Doppler spread. The dedicated REM for high-speed rail can be viewed as a spatial-temporal database consisting of the radio channel parameters along a given railway. The performance of the proposed Doppler spread compensation algorithms are evaluated with a typical OFDM-based broadband mobile system. Simulation results show that the link-level performance of high-speed rail broadband mobile communications can be improved significantly due to the REM-enabled radio channel condition awareness and the cognitive Doppler spread compensation algorithms. The REM-based cognitive radio approach presents a new paradigm for systems design of high-speed rail broadband mobile communications.
\end{abstract}

Keywords: Broadband mobile communication, Cognitive radio, Doppler spread compensation, High-speed rail, OFDM, Radio environment map (REM)

\section{Introduction}

High-speed rail is a type of passenger rail transport that operates significantly faster than the normal speed of rail traffic. Specific definitions by the International Union of Railways (UIC) and European Union include $200 \mathrm{~km} / \mathrm{h}$ for upgraded track and $250 \mathrm{~km} / \mathrm{h}$ or faster for new track [1]. High-speed rail receives considerable attention recently due to its cost-efficiency, almost all-weather operation, low carbon-dioxide $\left(\mathrm{CO}_{2}\right)$ emission, and many other advantages. In Japan, Shinkansen lines run at speeds of up to $300 \mathrm{~km} / \mathrm{h}$. In China, high-speed conventional rail lines operate at top speeds of $350 \mathrm{~km} / \mathrm{h}$ and reaches as high as $487.3 \mathrm{~km} / \mathrm{h}$ during a recent test run [1,2]. Research on even higher speed train in vacuum tube is also underway in China with target operational speed of $600-1000 \mathrm{~km} / \mathrm{h}$ in 10 years. Regional or international high-speed rail is under planning or construction in the United States, Europe, Asia, and South America [1]. Therefore, there is

\footnotetext{
* Correspondence: yozhao@bjtu.edu.cn

Institute of Broadband Wireless Mobile Communications, School of

Electronics and Information Engineering, Beijing Jiaotong University, Beijing,
} China an increasing yet challenging demand on broadband mobile communications for high-speed trains, as it is necessary to provide information (such as voice, Internet, video conference) and onboard entertainment services to passengers, support communication-based train control (CBTC), and transmit surveillance video and sensory data from the high-speed train to track-side base stations for the safety and efficient operation of high-speed rail. In addition, positive train control which has been developed in the United States is another major driver for highly reliable highspeed rail broadband mobile communications. Various technologies (such as leaky cable, GSM, satellite, and 802.11 WLAN) have been employed for railway wireless communications. However, each of the above-mentioned technologies has some shortcoming or limitations when applied to high-speed rail broadband mobile communications. The key problem associated with leaky cable is its high attenuation and high cost of deployment and maintenance; the key issue with GSM-based approach is its limited bandwidth and low data rate; the key problems associated with satellite-based approach are the lost of connection in tunnels, high systems cost, and significant 
delay; whereas the key problems with WLAN-based approach are the limited coverage per access point, modifications required to support smooth handover, and significant performance degradation for higher train speed (e.g., above $300 \mathrm{~km} / \mathrm{h})[3]$.

OFDM-MIMO-based long-term evolution (LTE) technology has been selected by UIC as the key technology for next generation wireless communication standard for railway (also known as "LTE-R") [4,5]. However, different from applying LTE to cellular communication networks, there are some new features for high-speed rail broadband mobile communications (as summarized in Table 1), which should be taken into consideration [6]. For example, the new high-speed rail line usually makes extensive use of viaducts, bridges, and tunnels. Therefore, the radio channel characteristics are quite different from those for cellular systems. Line-of-sight (LOS) path is usually available due to smaller cell size (typically, the cell radius of a track-side base station is less than $3 \mathrm{~km}$ ) and much higher antenna installed on top of the high-speed train (in contrast, the typical antenna height of a mobile station in cellular networks is about $1.5 \mathrm{~m}$ ). In addition, unlike mobile stations move randomly in cellular networks, the high-speed train has predictable positions and moving directions as it moves along a given course in a repetitive manner. Therefore, the radio channel conditions along a given highspeed rail line are predictable to some extent with the help of site-specific radio propagation prediction tools (e.g., utilizing the ray-tracing method) and three-dimensional (3D) geographical information system (GIS) databases.

Currently, there are mainly three approaches in literature to deal with Doppler spread and reduce the inter-subcarrier interference (ICI). The first approach is simply to estimate the dominant frequency offset $[7,8]$. Though many existing methods can estimate and remove the frequency offset quite accurately, their performance may degrade sharply under high-speed mobile scenarios with significant Doppler spread. The second approach basically employs signal processing and/or coding to reduce the sensitivity of the OFDM system to the frequency offset. For example, in $[9,10]$, time windowing has been introduced to reduce the ICI; another method known as the "ICI self-cancellation" has initially been proposed by Zhao and Haggman [11]; later on, Seyedi and Saulnier [12] proposed a new selfcancellation scheme with even better performance. In addition, an all phase OFDM system is proposed to improve the OFDM system's ability to resist frequency offset by employing all-phase FFT [13]. However, all of these methods have considerable computational complexity. In the third approach, sectorized antennas are utilized to divide the Doppler spectrum into a set of sub-spectra and thus reduces the time selectivity $[14,15]$. It copes well with large Doppler shift at the cost of increasing system complexity as well as hardware costs.

To mitigate the adverse impact of time-varying Doppler spread on OFDM-based broadband mobile communications, this article proposes cognitive Doppler compensation algorithms based on dedicated radio environment map (REM) for high-speed rail. The basic idea is to obtain fast and accurate channel condition awareness by retrieving the REM and then compensate the time-varying Doppler spread in a cost-efficient manner. The REM can be viewed as a spatial-temporal database that provides multi-domain environmental information (such as prior knowledge about the radio channel parameters and past experience) to cognitive radios [16-18]. Note that the term "cognitive radio" was first coined by Dr. Joseph Mitola III in late 1990s [19]. Over the past 10 years, cognitive radio has enabled a new design paradigm for wireless communications [20-22], mainly owing to its self-learning and situation-awareness capabilities. Compared with the approaches mentioned above, the REM-based cognitive Doppler compensation approach is cost-efficient and requires no additional hardware.

The rest of this article is organized as follows. In the following section, we first review the key challenges encountered by the high-speed rail broadband mobile communications, and then bring forward the system architecture of the proposed cognitive broadband mobile communication systems. In Section "REM-based cognitive Doppler spread compensation algorithms", two types of REM-based Doppler spread compensation algorithms are presented. The ICI due to Doppler spread is modeled for OFDM-based broadband mobile communications, which is instrumental for the development of cognitive Doppler spread compensation algorithms and link-level performance analysis. In Section "Link-level simulations of high-speed rail broadband mobile communications", the simulated performances of the proposed algorithms are discussed. Finally, we summarize this article in the last section.

Table 1 Key features of high-speed rail broadband mobile communications

\begin{tabular}{ll}
\hline Feature & Description \\
\hline Doubly selective channel & The high-speed rail radio channel is both time selective and frequency selective \\
$\begin{array}{ll}\text { Fast and frequent (group) handover } \\
\text { All passengers in the train move at the same direction and conduct handover almost simultaneously, resulting } \\
\text { high handover failure rate due to signaling storm and long process delay }\end{array}$ \\
$\begin{array}{l}\text { Predictable train location and } \\
\text { channel condition }\end{array}$ & $\begin{array}{l}\text { The high-speed rail train operates on a fixed course repetitively. This is in sharp contrast to traditional cellular } \\
\text { systems the mobile stations move randomly }\end{array}$ \\
\hline
\end{tabular}




\section{System overview of high-speed rail broadband mobile communications}

\section{Challenges of high-speed rail broadband mobile} communications

OFDM-MIMO is the key physical layer technology for next generation broadband mobile communications (e.g., 3GPP LTE), as it is immune to frequency-selective fading and narrow-band interference, and offers high spectral efficiency and capacity. However, as indicated by the highspeed rail channel coherent time (shown in Figure 1), with the increase of train speed and carrier frequency, the radio channel characteristics varies more rapidly and significantly along the high-speed rail line. As a result, doubly selective fading becomes even more serious. For example, supposing the carrier frequency is $3 \mathrm{GHz}$, when the train speed reaches $360 \mathrm{~km} / \mathrm{h}$, the maximum Doppler frequency shift is as high as $1 \mathrm{kHz}$. The resultant ICI could degrade the OFDM system performance dramatically. It is critical to estimate the channel quickly and accurately to ensure the performance of OFDM-MIMO-based communication systems [23-25].

Though the channel estimation problems for high-speed rail have been investigated by some researchers, there is no ideal solution yet. Some proposed algorithms are too sophisticated (with heavy computational load) to meet the requirements of real-time implementation [26]; some proposed algorithms simply could not produce good enough results [27, and the references therein]. For example, Liu et al. [27] proposed a channel estimation algorithm based on comb-pilot. This algorithm works well under time- invariant multipath channel. However, it cannot work well under fast time-variant channel (e.g., using COST 259 RA channel model) due to the ICI. There exists an error floor for the minimum square error of channel estimation and the bit error rate (BER) even if the signal-to-noise ratio (SNR) is very high [27]. Hardware-in-the-loop simulation also shows that the performance degrades significantly under Rayleigh fading channels when the train speed reaches $350 \mathrm{~km} / \mathrm{h}$ [28].

Note that prior knowledge about high-speed rail channel characteristics has not carefully been exploited for fast channel estimation yet. Considering the repetitive movement of a high-speed train along a given high-speed rail line, the radio channel characteristics could be predictable (to some extent) based on the location of high-speed train, local terrain information from a GIS database and past experience. It is highly possible to exploit such prior knowledge and improve the performance of high-speed rail broadband mobile communication systems by eliminating or reducing the error floor in channel estimation and conducting more effective Doppler compensation thereafter.

\section{System architecture of cognitive broadband mobile communications}

The proposed system architecture of cognitive high-speed rail broadband mobile communication systems is shown in Figure 2. Considering the high penetration loss (typically $20 \mathrm{~dB}$ ) of the high-speed train [6], it is assumed that a cognitive wireless gateway is developed and installed on the high-speed train to support various types of radio

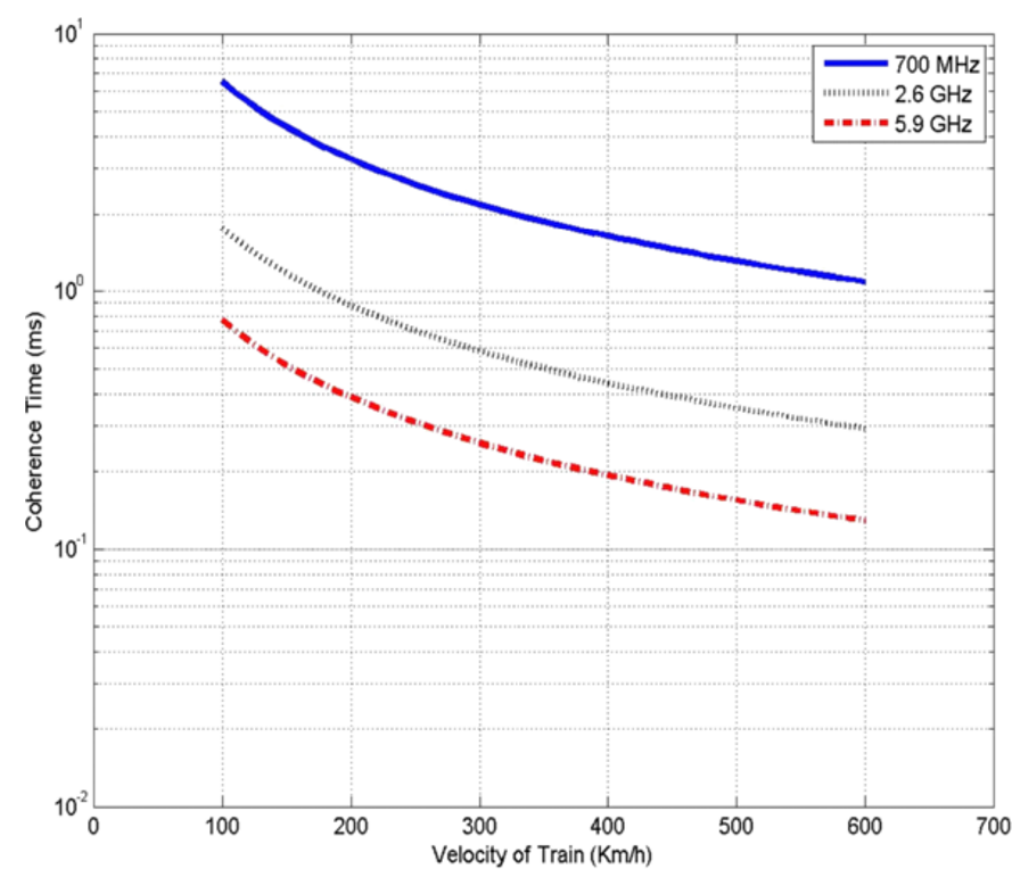

Figure 1 Coherence time for high-speed rail mobile communications. 


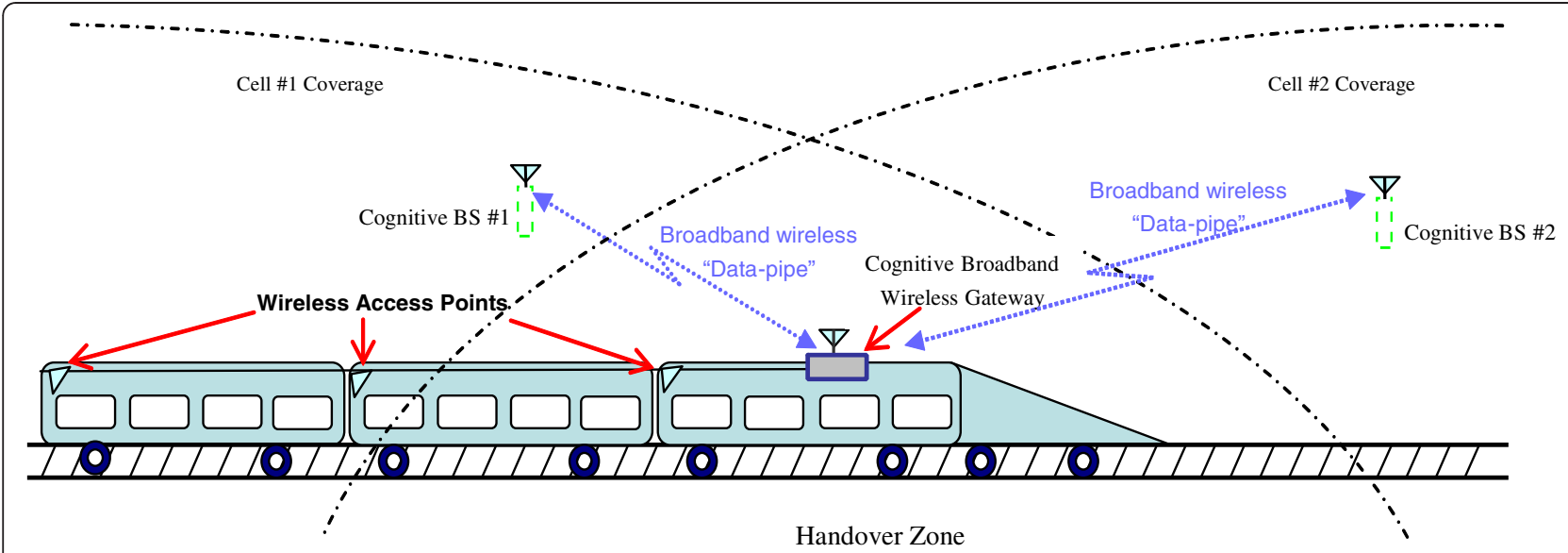

Figure 2 Architecture of cognitive high-speed rail mobile communication systems based on a dedicated REM for railway.

devices through the distributed multi-mode access points inside the train. In this way, all communication traffic to/from different access points is multiplexed/demultiplexed through the broadband mobile gateway. A broadband mobile "data pipe" (e.g., based on the 3GPP LTE standard) is set up between the cognitive wireless gateway and the cognitive track-side base stations (BS).

\section{Dedicated REM for high-speed rail}

The main information elements of a dedicated REM for high-speed rail include the following: channel parameters such as the number of paths, channel coefficients, delay, and angle of arrival for each path at a given position along the high-speed rail line (as illustrated in Table 2), and the local spectrum policy and spectrum usage information, etc. Note that the channel parameters for a given section of high-speed railway can be initially populated into the REM via off-line site-specific channel prediction tools (e.g., based on 3D ray-tracing and GIS database) or prior field measurement with channel sounding equipment, and further updated or fine-tuned so that the performance of high-speed rail broadband mobile communications can be optimized along with the repetitive movement of highspeed train over a given course [29]. By retrieving the dedicated REM for high-speed rail, fast channel awareness can be obtained. With the feedback on link performance, cognitive broadband mobile communication systems can update or fine-tune the channel parameters while the high-speed train moves along the given course repetitively.

\section{Positioning accuracy of the high-speed train}

Accurate positioning and speed measurement of the high-speed train is critical for the proposed REM-based cognitive Doppler spread compensation algorithms, as accurate location information is the prerequisite to look up the dedicated REM for HSR and obtain the situation awareness. Odometer is the most widely used in train positioning system because of its low cost and simpleto-implement properties. However, the performance of train odometer-based positioning would easily be influenced by the accumulated counting error due to idling or skidding and the wheel diameter attrition. Integrated train positioning algorithms are developed to leverage both GPS and odometer [30]. Furthermore, the position and speed of the high-speed train can also be periodically calibrated with the balise, which is an electronic beacon or transponder placed between the rails of a railway as part of an automatic train protection system. State-of-the-art train speed and position measurement method such as multi-sensor information fusion in CBTC systems enables very high location accuracy (on the order of $10^{-2} \mathrm{~m}$ ) [31]; for the high-speed maglev train driven by the long-stator

Table $\mathbf{2}$ Key information elements in the dedicated REM for high-speed rail

\begin{tabular}{|c|c|c|c|}
\hline Index & Chann & & \\
\hline Record \#1 & Numbe & & \\
\hline Train-top antenna location \#1 & Chann & & \\
\hline Timestamp \#1 (time, date, day of the week) & $a_{0}$ & $\varphi_{0}$ & $\tau_{0}$ \\
\hline & $\ldots$ & $\ldots$ & $\ldots$ \\
\hline & $a_{N p 1-1}$ & $\varphi_{N p 1-1}$ & $\tau_{N p 1-1}$ \\
\hline Record \#2 & Numbe & & \\
\hline$\ldots$ & $\ldots$ & & \\
\hline
\end{tabular}


synchronous linear motor, even higher location accuracy (with the positioning resolution of $4.3 \mathrm{~mm}$ ) has been achieved in the lab measurement by using another method based on measuring cog-slots of the long-stator [32]. In addition, other positioning methods (such as RF fingerprinting [33]) can also be employed as complementary approaches.

\section{REM-based cognitive Doppler spread compensation algorithms}

The proposed high-speed rail mobile communication systems can obtain channel awareness simply by looking up the dedicated REM for railway, instead of running complicated channel estimation algorithms. The retrieved channel parameters can be exploited by the Doppler spread compensation algorithms at the receiver. In this section, the REM-based Doppler spread compensation algorithms and the analytical performance are presented.

We first derive the mathematics model for both the desired signal and the ICI due to Doppler spread with an OFDM symbol. Then cognitive Doppler compensation algorithms are developed to mitigate or eliminate the ICI.

The baseband OFDM symbol with cyclic prefix $(\mathrm{CP})$ is modeled as

$$
S(k)=\frac{1}{\sqrt{N}} \sum_{i=0}^{N-1} d_{i} e^{j \frac{2 \pi}{N} i k}, k=-L_{g}, \cdots, 0, \cdots N-1
$$

where $N$ is the number of sub-carriers, $L_{g}$ is the discrete length of the $\mathrm{CP}$, and $d_{i}$ is the transmitted symbol on the $i$ th subcarrier.

The time-variant impulse response of a wireless channel is defined by

$$
h(t, \tau)=\sum_{n=0}^{N_{p}-1} \alpha_{n} t e^{j \phi_{n}(t)} e^{j 2 \pi \Delta f_{n}(t) t} \delta\left(\tau-\tau_{n}(t)\right)
$$

where $N_{p}$ is the number of the detected paths between the track-side BS and the wireless gateway on the train, $\alpha_{n}, \Phi_{n}, \Delta f_{n}$, and $\tau_{n}$ are the averaged fading amplitude, phase shift, Doppler frequency shift, and time delay corresponding to the $n$th path, respectively. For the highspeed rail radio channel, we assume that these parameters are time-invariant in the duration of an OFDM symbol, and $\Phi_{n}=0$. Then the channel impulse response (CIR) is determined by

$$
h(t, \tau)=\sum_{n=0}^{N_{p}-1} \alpha_{n} e^{j 2 \pi \Delta f_{n} t} \delta\left(\tau-\tau_{n}\right)
$$

The received baseband signal $r(t)$ is modeled as the convolution of $S(t)$ with the CIR $h(t, \tau)$ plus a complex additive white Gaussian noise $n(t)$, i.e.,

$$
\begin{aligned}
r(t) & =\int S(t-\tau) h(t, \tau) d \tau+n(t) \\
& =\sum_{n=0}^{N_{p}-1} \alpha_{n} e^{2 \pi \Delta f_{n} t} S\left(t-\tau_{n}\right)+n(t)
\end{aligned}
$$

where $t \in\left[-T_{g}, T\right], T_{\mathrm{g}}$ is the duration of the $\mathrm{CP}$ section in an OFDM symbol, and $T$ is the duration of the data section.

After sampling the received signal at a sampling rate of $1 / T_{s}$, the discrete received signal is

$$
r(m)=\sum_{n=0}^{N_{p}-1} \alpha_{n} e^{j 2 \pi \Delta f_{n} m T_{s}} S\left(m-p_{n}\right)+n(m)
$$

where $m=-L_{g}, \ldots, 0,1, \ldots, N-1$, and

$$
\begin{aligned}
& p_{n}=\left\lceil\frac{\mathrm{\tau}_{n}}{T_{s}}\right\rceil, \\
& 0=\mathrm{\tau}_{0}<\mathrm{\tau}_{1}<\mathrm{\tau}_{2}<\ldots<\mathrm{\tau}_{N_{p}-1}
\end{aligned}
$$

To demodulate the OFDM signal, the received signal goes through an FFT. Inter-symbol interference (ISI) can be avoided by selecting the guard interval such that $T_{g}>\tau_{N p-1}$ is satisfied. Assuming perfect time synchronization, the received signal after removing the guard interval and FFT operation is depicted as

$$
R(l)=\frac{1}{\sqrt{N}} \sum_{m=0}^{N-1} r(m) e^{-j \frac{2 \pi}{N} m l}
$$

where $l=0,1,2, \ldots, N-1$

Finally, $R(l)$ is calculated by

$$
R(l)=\frac{1}{N} \sum_{n=0}^{N_{p}-1} \sum_{i=0}^{N-1} d_{i} \sum_{m=0}^{N-1} \alpha_{n} e^{i 2 \pi\left(\Delta f_{n} T_{s}+\frac{i-l}{N}\right) m} e^{-j \frac{2 \pi}{N} p_{n} i}+n(l)
$$

When supposing $i=l$, the desired signal is derived:

$$
R_{S}(l)=d_{l} H(l),
$$

where the channel gain is

$$
H(l)=\frac{1}{N} \sum_{n=0}^{N_{P}-1} \alpha_{n} e^{-j \frac{2 \pi}{N} P_{n l} l} e^{j \pi(N-1) \Delta f_{n} T_{s}} \frac{\sin N \pi \Delta f_{n} T_{s}}{\sin \pi \Delta f_{n} T_{s}}
$$

When supposing $i \neq l$, the ICI at the $l$ th subcarrier is derived as follows:

$$
\begin{gathered}
R_{I}(l)=\frac{1}{N} \sum_{i=0, i \neq l}^{N-1} d_{i} \sum_{n=0}^{N_{p}-1} \alpha_{n} e^{-j \frac{2 \pi}{N} P_{n} i} e^{j \pi(N-1)\left(\Delta f_{n} T_{s}+\frac{i-l}{N}\right)} \\
\frac{\sin N \pi\left(\Delta f_{n} T_{s}+i-l\right)}{\sin \pi\left(\Delta f_{n} T_{s}+\frac{i-l}{N}\right)}
\end{gathered}
$$

Therefore, the SIR for the $l$ th sub-carrier is depicted by 


$$
\operatorname{SIR}(l)=\frac{P_{S}(l)}{P_{I}(l)}=\frac{\left|R_{S}(l)\right|^{2}}{\left|R_{I}(I)\right|^{2}}
$$

\section{REM-based cognitive Doppler spread compensation algorithms}

In this section, a simple Doppler spread compensation algorithm is first designed using the Doppler frequency shift information stored in the dedicated REM for high-speed rail. Then a more advanced Doppler spread compensation algorithm is developed by fully utilizing channel information from the REM. First of all, we redefine the symbols to be used in this section: $N_{p}$ is the number of detectable paths in the high-speed rail radio channel, and $\alpha_{n}, \Delta f_{n}, p_{n}$ are the fading amplitude, Doppler frequency shift, and discrete time delay in the $n$th path, respectively $\left(0 \leq n \leq N_{p}-1\right)$. Note that in this section, the time-discrete transmitted OFDM signal is $s(m)$, which is usually composed of a number of consecutive OFDM symbols, and $z(m)$ is additive Gaussian white noise while $r(m)$ is the received signal sequence.

Note that the REM-based cognitive Doppler spread compensation algorithm is employed on $r(m)$, which is obtained after the A/D conversion of the time-continuous received baseband signal $r(t)$. It exploits the channel parameters stored in the dedicated REM for railway to preprocess the received signal sequence so as to eliminate or mitigate the multipath effects of the wireless channel.

\section{Simple Doppler compensation algorithm (Algorithm 1)}

Algorithm 1 aims to estimate the average Doppler frequency shift and then compensate it directly. Since the signal power for each path is different, the path with higher signal power has more significant impact on the average Doppler frequency shift of the received signal and has a higher weight in the average Doppler frequency shift. The algorithm is depicted as follows.

The Doppler frequency shift to be compensated is estimated in a weighted sum manner as follows:

$$
\Delta f_{\text {comp }}=w_{0} \Delta f_{0}+w_{1} \Delta f_{1}+\cdots+w_{N_{p}-1} \Delta f_{N_{p}-1}
$$

where

$$
\Delta f_{n}=\frac{v}{\lambda} \cos \phi_{n}\left(n=0, \cdots, N_{p}-1\right)
$$

where $v$ is the velocity of the train relative to the BS, $\lambda$ is the wave length of the central carrier frequency, $\phi_{n}$ is the angle between the moving direction of the highspeed train and the reverse direction of the $n$th incident wave, as illustrated in Figure 3; and the weight for the $n$th path $\left(w_{n}\right)$ is proportional to the SNR of the $n$th path while satisfying

$$
\sum_{n=0}^{N_{p}-1} w_{n}=1
$$

Then, the received signal sequence after Doppler compensation can be expressed as

$$
r_{\text {comp }}(m)=r(m) e^{-j 2 \pi \Delta f_{c o m p} m T_{s}}
$$

where $T_{s}$ is the sampling interval.

\section{Advanced Doppler spread compensation algorithm (Algorithm 2)}

In this algorithm, we employ an iterative method to extract the signal of the first path based on the fact that different paths are distinguishable in their arriving time

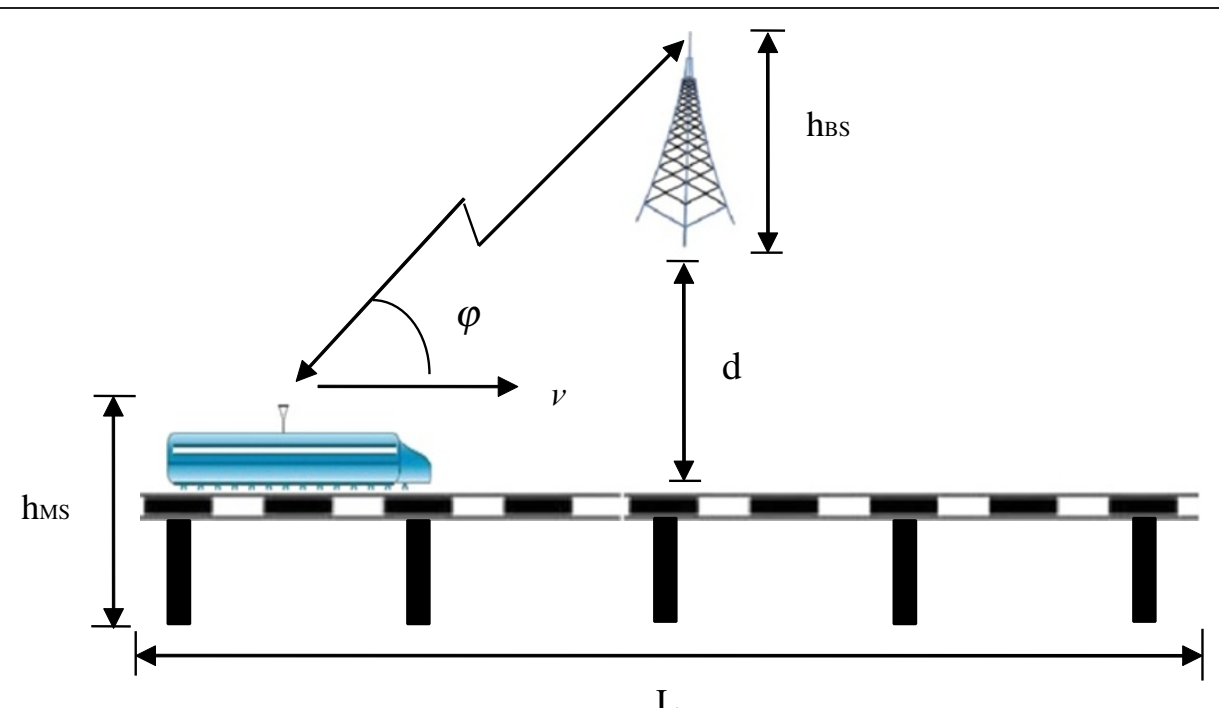

Figure 3 Simulated LOS scenarios for high-speed rail broadband wireless communication, (a high-speed train moves on a viaduct). 


$$
s(m)=\left\{\begin{array}{l}
r(m) /\left(\alpha_{0} e^{j 2 \pi \Delta f_{0} m T_{s}}\right), 0 \leq m<p_{1} \\
{\left[r(m)-\alpha_{1} e^{j 2 \pi \Delta f_{1} m T_{s}} s\left(m-p_{1}\right)\right] /\left(\alpha_{0} e^{j 2 \pi \Delta f_{0} m T_{s}}\right), p_{1} \leq m<p_{2}} \\
{\left[r(m)-\alpha_{2} e^{j 2 \pi \Delta f_{2} m T_{s}} s\left(m-p_{2}\right)-\alpha_{1} e^{2 \pi \Delta f_{1} m T_{s}} s\left(m-p_{1}\right)\right] /\left(\alpha_{0} e^{j 2 \pi \Delta f_{0} m T_{s}}\right), p_{3} \leq m<p_{4}} \\
\cdots \\
{\left[r(m)-\alpha_{N_{p}-1} e^{j 2 \pi \Delta f_{N_{p}-1} m T_{s}} s\left(m-p_{N_{p}-1}\right)-\ldots-\alpha_{1} e^{j 2 \pi \Delta f_{1} m T_{s}} s\left(m-p_{1}\right)\right] /\left(\alpha_{0} e^{j 2 \pi \Delta f_{0} m T_{s}}\right), p_{N_{p}-1} \leq m \leq M-1}
\end{array}\right.
$$

as illustrated in Figure 4. For example, from $t_{0}$ to $t_{1}$, the received signal contains the first path's signal only, and from $t_{1}$ to $t_{2}$, the received signal is the combination of the first path's signal and the second path's signal. By employing the channel parameters stored in the REM for railway, the transmitted signal can be recovered in an iterative approach with the following algorithm.

The received signal sequence $r(m)$ can be expressed in another form:

$$
\sum_{n=0}^{k} \alpha_{n} e^{j 2 \pi \Delta f_{n} m T_{s}} s\left(m-p_{n}\right)+z(m)=r(m)
$$

for $\quad 0 \leq k \leq N_{p}-2, p_{k} \leq m<p_{k+1}$, for $\quad k=N_{p}-1, p_{N p_{-1}} \leq m$ $\leq M-1$, where $0 \leq m \leq M-1$, and $M$ is the length of the transmitted signal sequence.

From (17), we can get $M$ linear equations with $M$ unknowns. Supposing that perfect channel parameters are available (from the REM for railway) and the SNR is high enough that the additive noise added to the channel is negligible, $s(m)$ can be derived by solving these equations and expressed by (18). In this way, the ICI resulting from Doppler spread can be eliminated or mitigated systematically.

In practice, $r(m)$ in (17) and (18) should be considered in the context of a time division duplex (TDD) or frequency division duplex (FDD) "frame" of an OFDM communication system, consisting of a number of OFDM symbols as illustrated in Figure 5.

For the TDD OFDM systems, downlink frames and uplink frames are transmitted alternatively in time. For example, in the downlink communication, the time duration of guard period and uplink frame (as shown in Figure 5) can actually serve as the "guard interval" (in which there is no RF emission from the base station). Therefore, even with the maximum path delay in the high-speed rail mobile communication environment, the samples of the previous frame will not interfere with the samples of the current frame. So, the advanced Doppler spread compensation algorithm is especially good for TDD OFDM communication systems. The iterative approach in (18) can be employed to each "frame" of a TDD OFDM system.

For FDD OFDM systems, in order to employ this advanced Doppler spread compensation algorithm, a guard

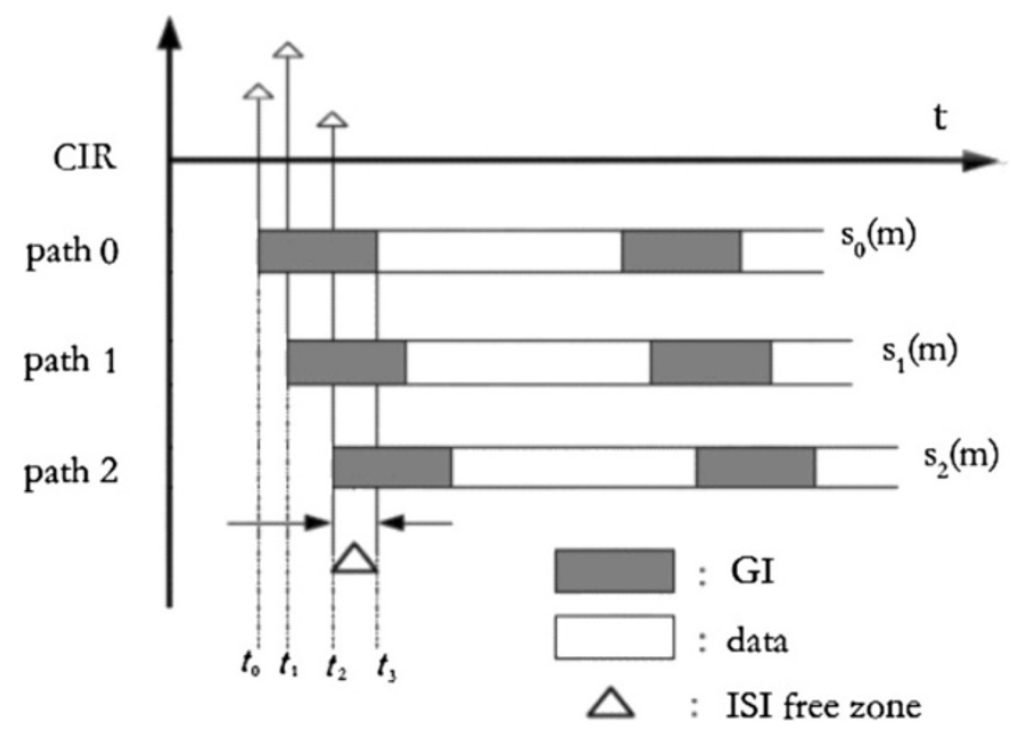

Figure 4 Multi-path propagation of OFDM signals. 


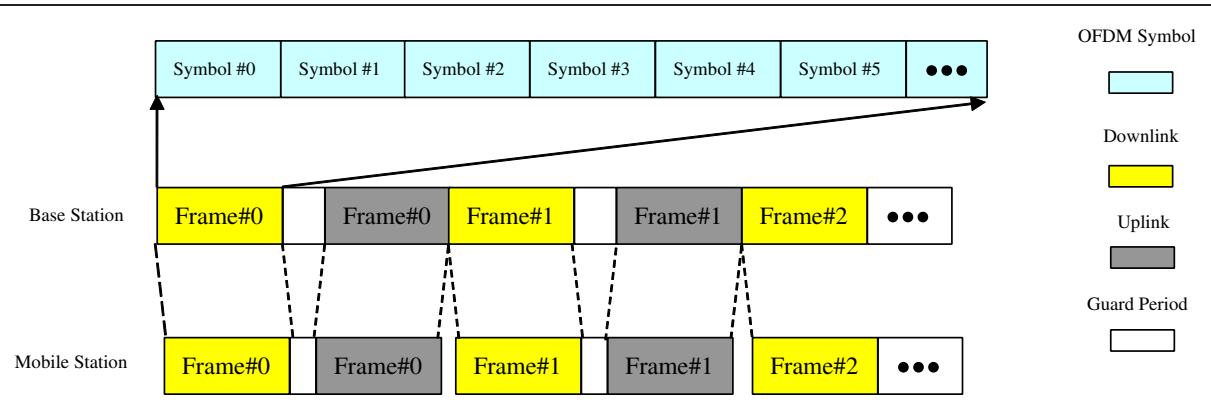

(A)

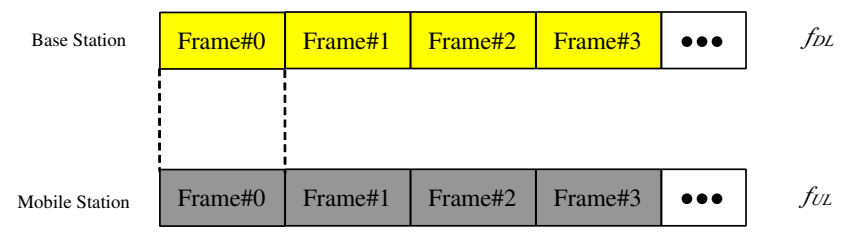

(B)

Figure 5 Frame structure of a typical OFDM communication system. (A) TDD. (B) FDD.

period (i.e., a pure empty prefix) needs to be inserted at the beginning of each frame.

\section{Analytical performance analysis}

For the channel model described in (17), the samples of the observed signal at the receiver can be modeled by the following linear model:

$$
H \times S+Z=R
$$

where $S$ is an $M \times 1$ vector consisting of $M$ samples of the observed signal at the receiver, $H$ is the $M \times M$ observation matrix (i.e., channel matrix), $Z$ is a $M \times 1$ noise vector consisting of $M$ random variables following the zero-mean normal Gaussian distribution (with variance of $\sigma^{2}$ ).

$$
\begin{aligned}
& {\left[\begin{array}{lll}
h_{0,0} & & \\
h_{1,0} & h_{1,1} & \\
h_{2,0} & h_{2,1} & h_{2,2} \\
\ldots \ldots & \\
h_{M-1,0} & h_{M-1,1} \ldots \ldots h_{M-1, M-1}
\end{array}\right] \cdot\left[\begin{array}{l}
s(0) \\
s(1) \\
s(2) \\
\ldots \ldots \\
s(M-1)
\end{array}\right]} \\
& +\left[\begin{array}{l}
z(0) \\
z(1) \\
z(2) \\
\ldots \ldots \\
z(M-1)
\end{array}\right]=\left[\begin{array}{l}
r(0) \\
r(1) \\
r(2) \\
\ldots \ldots \\
r(M-1)
\end{array}\right]
\end{aligned}
$$

where

$$
h_{i, i-p_{n}}=\alpha_{n} e^{j 2 \pi \Delta f_{n} i T_{S}}
$$

The minimum-variance unbiased estimator of $S$ is an efficient estimator, which obtains the Cramer-Rao lower bound of $\sigma^{2}\left(H^{T} H\right)^{-1}$ [34].

For a two-path channel model, the minimum variance of estimation error for the $M$ samples of the received signal can be calculated by diag $\left(H^{T} H\right)^{-1}$ and plotted in Figure 6.

As can be seen from Figure 6, when there is a dominant path (e.g., $\alpha_{1}=0.1 \alpha_{0}$ ), the variance of sample estimation errors are close to $\sigma^{2}$; whereas when there is no dominant path (e.g., $\alpha_{1}=0.9 \alpha_{0}$ ), the variance of sample estimation errors could be as high as $5 \sigma^{2}$. Accordingly, with the noise power shown in Figure 6, the analytical BER performance of the advanced Doppler spread compensation algorithm (Algorithm 2) is shown in Figure 7.

\section{Link-level simulations of high-speed rail broadband mobile communications}

This section presents the simulated link-level performance of an OFDM-based high-speed rail broadband mobile communication system.

\section{Assumptions and system parameters}

As a case study example, TD-LTE (i.e., the TDD version of LTE) standard is adopted for the broadband mobile data-pipe between the cognitive wireless gateway on the train and the track-side base station. The system parameters for TD-LTE are summarized in Table 3.

For the TD-LTE frame structure, each frame consists of ten subframes while each subframe contains two slots. Each slot is $0.5 \mathrm{~ms}$ long, consisting of seven OFDM symbols. In our simulations, for the sake of simplicity, the 


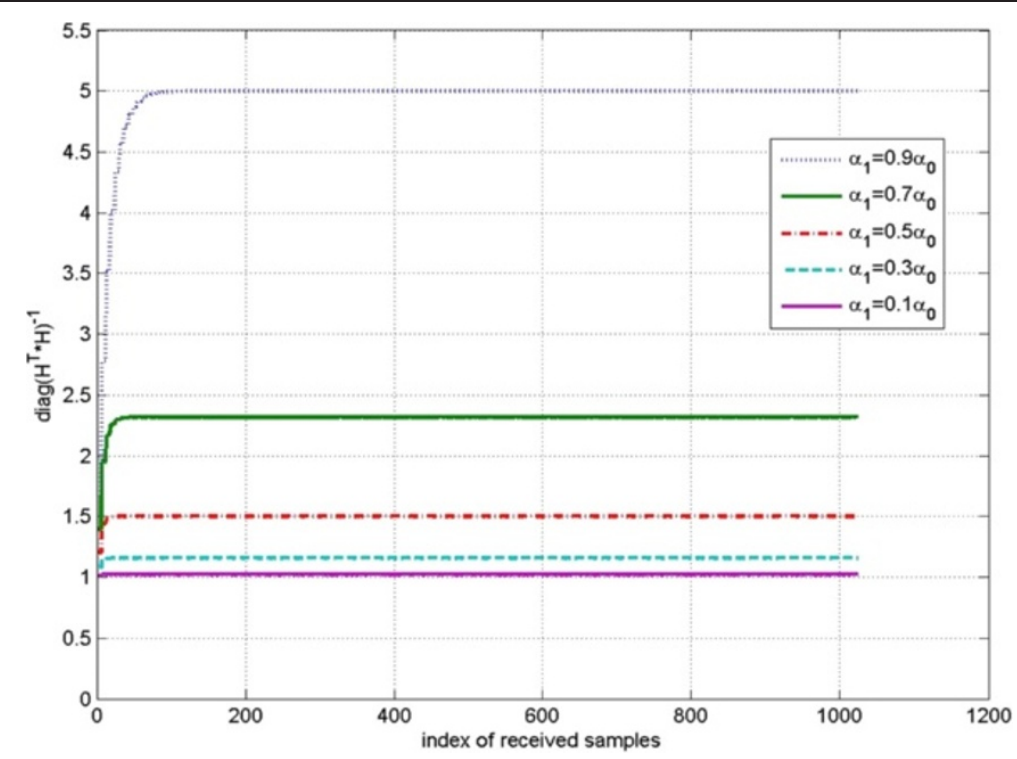

Figure 6 Minimum variances of the sample estimation error.

length of CP in each OFDM symbol is set as 72 samples. Figure 8 shows the reference signal pattern of TD-LTE.

\section{Simulation methodology and simulation scenarios}

To evaluate the performance of a cognitive high-speed rail broadband mobile communication system, dynamically changing channel conditions, including both LOS and non-LOS (NLOS) scenarios, need to be simulated.
Figure 9 shows the dynamic channel emulator employed in our simulation. Various radio channel conditions due to doubly selective fading can be generated through this dynamic channel emulator.

For the sake of simplicity, an abstract two-path channel model is used in our simulation, while it is fairly straightforward to extend it to more generic channel models with multiple paths. The number of paths, pathloss coefficient, time delay, Doppler frequency shift, and

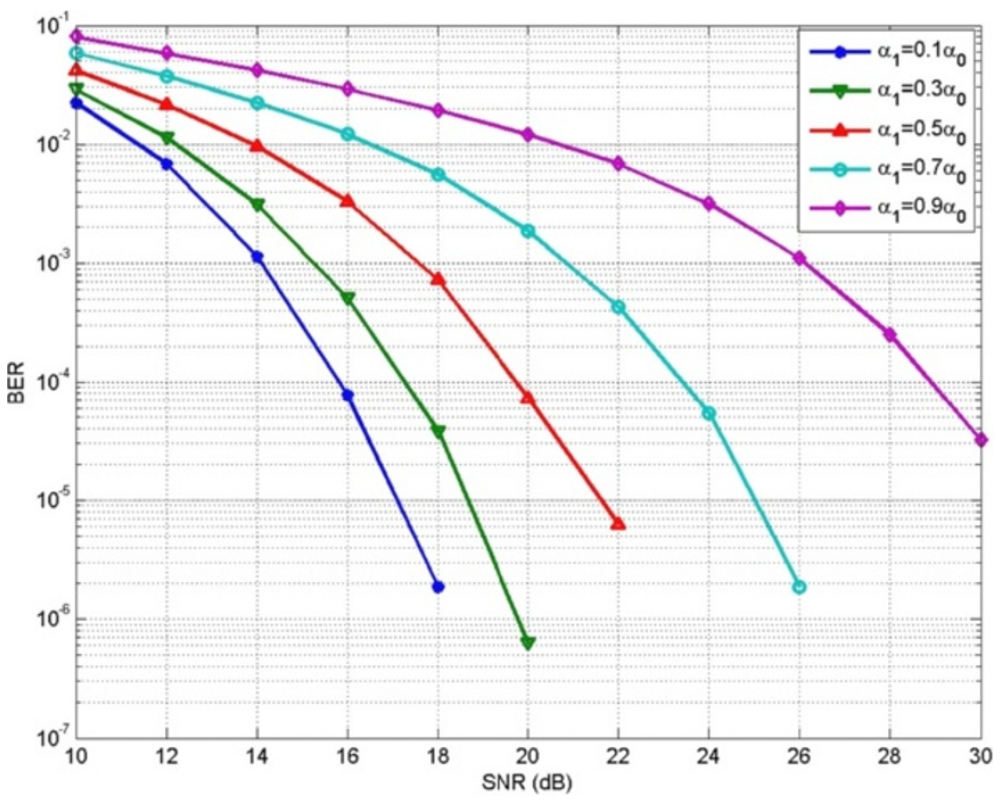

Figure 7 Analytical BER performance versus channel conditions (assuming a two-path channel model; train speed: $240 \mathrm{~km} / \mathrm{h}$ ). 
Table 3 TD-LTE downlink parameters

\begin{tabular}{ll}
\hline Parameters & Values \\
\hline Transmission bandwidth & $10 \mathrm{MHz}$ \\
Sub-carrier spacing & $15 \mathrm{kHz}$ \\
Frame length & $10 \mathrm{~ms}$ \\
Sampling frequency & $15.36 \mathrm{MHz}$ \\
FFT size & 1024 \\
Number of data sub-carriers & 601 (including DC sub-carrier) \\
OFDM symbol duration & $66.67 \mu \mathrm{ss}$ \\
Total symbol time duration & $71.4 \mu \mathrm{s}$ (normal CP) \\
Symbols per slot & 7 \\
Number of protection sub-carriers & 212 (left side), 211 (right side) \\
Modulation schemes & $16 \mathrm{QAM}$ \\
Carrier frequency & $2.6 \mathrm{GHz}$ \\
\hline
\end{tabular}

the noise level for each path are the main parameters stored in the dedicated REM for high-speed rail.

\section{Scenario type 1: LOS scenarios}

As depicted by Figure 3, LOS scenarios are the most common scenarios for high-speed rail broadband mobile communication, as the cell radius of track-side base stations is usually less than $3 \mathrm{~km}$ and extensive use of viaducts and bridges along the high-speed rail line.

The parameters assumed in the LOS scenario are summarized in Table 4. Note the height of high-speed train is about $2 \mathrm{~m}$ and the viaduct is $10 \mathrm{~m}$ above the ground.

For the LOS scenario, the SNR at the cell edge is assumed as $20 \mathrm{~dB}$, and the LOS path loss (PL) between the wireless gateway and the track-side base station follows the WINNER II D2a path-loss model [35], which is expressed as follows

$$
P L=P L_{D 1}+P L_{e n v}
$$

where

$\mathrm{PL}_{D 1}$ (the basic path-loss) can be expressed as

$$
\boldsymbol{P} \boldsymbol{L}_{D 1}=\left\{\begin{array}{c}
44.2+21.5 \log _{10} d+20 \log _{10}(0.2 f) \\
+X_{\sigma} \quad 30 \boldsymbol{m} \leq d<d_{b p} \\
44.2+21.5 \log _{10} d_{b p}+40 \log _{10}\left(\frac{d}{d_{b p}}\right) \\
+20 \log _{10}(0.2 f)+X_{\sigma} \quad d \geq d_{b p}
\end{array}\right.
$$

and

$$
\boldsymbol{P L}_{\text {env }}=\left\{\begin{array}{cl}
0 & d \leq d_{\boldsymbol{B P}}^{\prime} \\
18.5 \log _{10}\left(\frac{d}{d_{B \boldsymbol{P}}^{\prime}}\right) & d>d_{B \boldsymbol{P}}^{\prime}
\end{array}\right.
$$

where $f$ is the carrier frequency (in $\mathrm{GHz}$ ), $X_{\sigma}$ is a zeromean Gaussian distributed random variable (in $\mathrm{dB}$ ) with standard deviation $(\sigma)$, and $d$ is the distance between track-side BS and the wireless gateway on the train (MS).

$$
d_{b p}=4 h_{B S} h_{M S} f / c
$$

where $h_{\mathrm{BS}}$ is the antenna height of the track-side base station and $h_{\mathrm{MS}}$ is the antenna height of the wireless gateway on the train; $c$ is the velocity of light in vacuum. $d_{\mathrm{BP}}^{\prime}$ is the environment break-point length, which is set as $120 \mathrm{~m}$ (which is different from $d_{\mathrm{bp}}$ ).

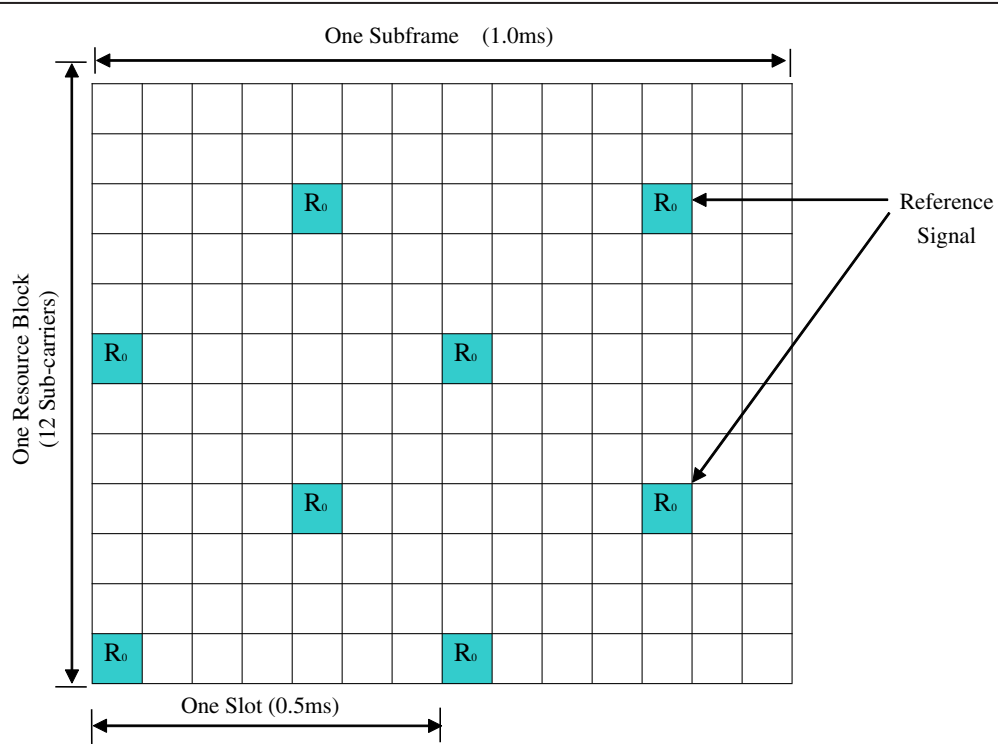

Figure 8 Reference signal pattern inside a TD-LTE subframe. 


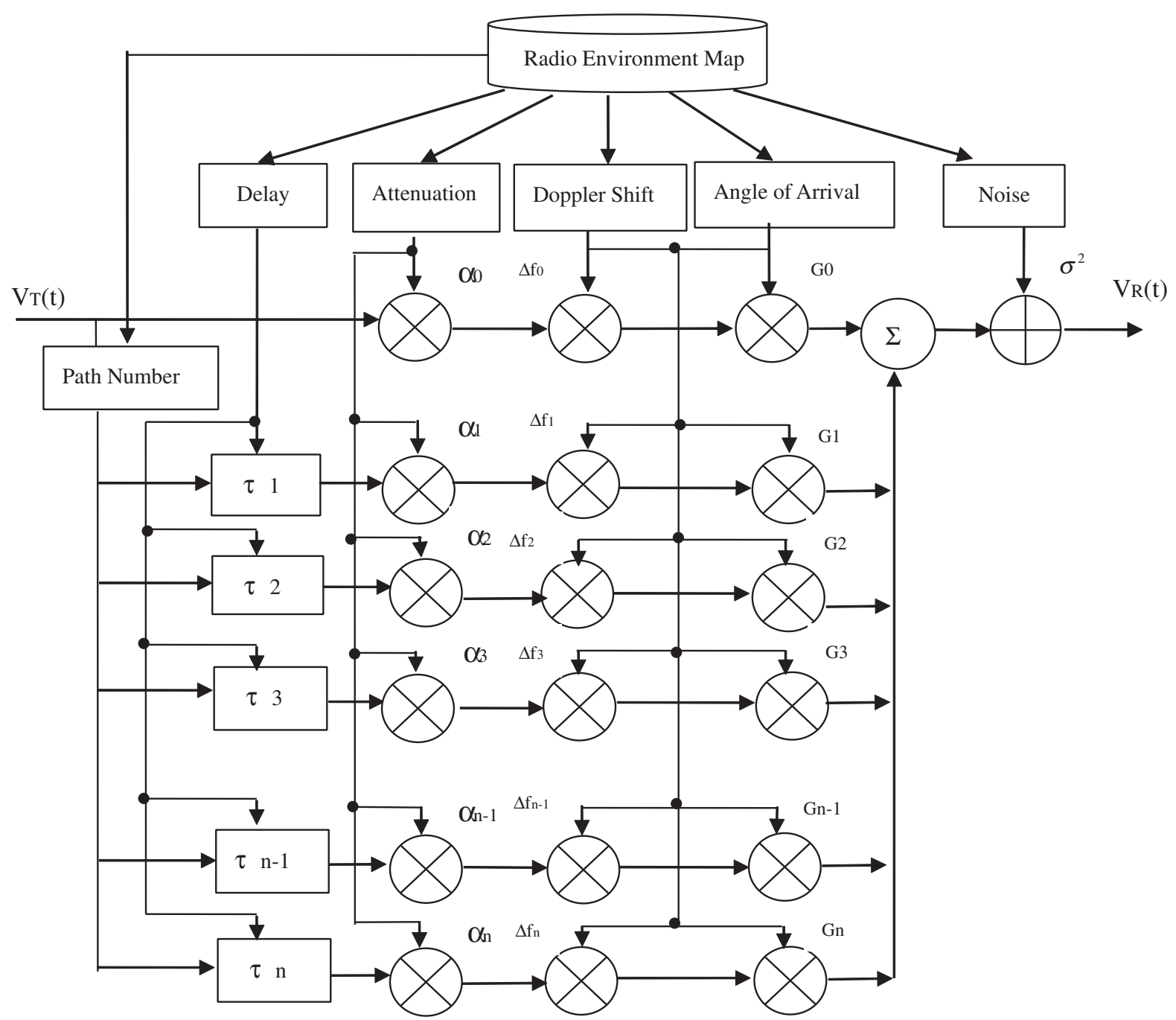

Figure 9 Block diagram of REM-based dynamic channel emulator employed in the simulation $\left(\tau_{n}, a_{n}, \Delta f_{n}\right.$, and $G_{n}$ are the time delay, channel coefficient, Doppler frequency shift, antenna gain for the $n$th path, respectively; and $\sigma^{2}$ is the white Gaussian noise level).

For the high-speed rail LOS scenarios, it is assumed that the signal power of the second path is at least $10 \mathrm{~dB}$ weaker than that of the dominant LOS path; with regarding to the LOS path, the time delay of the second path varies from 65 ns to $1.3 \mu$ s (according to the WINNER II D2a model); the angle of arrival for the first path is determined according to the position of the wireless gateway antenna on the train and the position of track- side base station, whereas the angle of arrival for the second path varies from 0 to $2 \pi$.

\section{Scenario type 2: NLOS scenarios}

The link-level performance is also simulated under the NLOS scenarios where LOS path is either blocked by buildings/hills or shadowed by foliages. Rayleigh fading is assumed for NLOS scenarios, where the signal

\section{Table 4 Parameters for the LOS scenario}

\begin{tabular}{ll}
\hline Parameter & Value \\
\hline Travel distance of high-speed train $(L)$ & $5000 \mathrm{~m}$ \\
Speed of train & $240 \mathrm{~km} / \mathrm{h}$ \\
Distance between track-side base station antenna and high-speed rail $(d)$ & $75 \mathrm{~m}$ \\
Antenna height at base station $\left(h_{\mathrm{BS}}\right)$ & $24 \mathrm{~m}$ \\
Antenna height on top of high-speed train $\left(h_{\mathrm{MS}}\right)$ & $12 \mathrm{~m}(=2 \mathrm{~m}+10 \mathrm{~m})$
\end{tabular}


strength of each path follows the Rayleigh distributions $[36,37]$. Time delay for the second path still follows a uniform distribution between $65 \mathrm{~ns}$ and $1.3 \mu \mathrm{s}$. The angle of arrival for the first path follows a normal distribution with the mean value same as that for the LOS scenario (as if the LOS path were not blocked), whereas the angle of arrival for the second path follows a uniform distribution between 0 and $2 \pi$.

\section{Simulation results and analysis \\ Simulation results and analysis for LOS scenarios}

Figure 10 shows the Doppler frequency shift vs. time and variation rate of Doppler shift versus time for the LOS path signal when the high-speed train moves on a viaduct of $5000 \mathrm{~m}$ long at a constant speed of $240 \mathrm{~km} / \mathrm{h}$ as depicted in Figure 9. A rapid Doppler shift variation (as high as $500 \mathrm{~Hz} / \mathrm{s}$ ) is observed when the high-speed train passes by the track-side BS.

The simulated BER performance under the LOS scenario is shown in Figure 11. The cognitive Doppler compensation algorithms support adaptive Doppler compensation by exploiting the channel information from the REM for highspeed rail. For comparison purpose, the BER performance of an OFDM receiver without Doppler compensation is also simulated. As indicated by Figure 11, cognitive approach, especially the advanced Doppler compensation algorithm, results in significant performance gain (i.e., much lower
BER) over the approach without Doppler compensation. Note that the approach without Doppler compensation can only work well when the train passes by the track-side base station, as the Doppler frequency shift of the dominant (LOS) path is negligible during this very short time period, whereas the BER is lower than $10^{-7}$ for most of the time (i.e., from 15 to 60 s) by exploiting the advanced Doppler compensation algorithm (marked as Algorithm 2 in this figure).

The BER performance under LOS scenario is further simulated under two different train speeds. As shown in Figure 12, supposing perfect channel information are employed by looking up the REM database, the degradation of link performance when the train moves faster (at $360 \mathrm{~km} / \mathrm{h}$ ) is negligible when adopting the advanced cognitive Doppler compensation algorithm.

In practice, considering the additive noise imposed to the channel sounding systems, it is inevitable that the channel information (such as the pathloss coefficient, delay, and angle of arrival for each path) stored in the REM is different from the true value due to the estimation error under a given SNR [34]. By comparing Figures 12 and 13, we can see that when imperfect channel information is employed, the BER performance degrades; the higher the train speed, the larger the degradation. In this simulation, random channel estimation errors, complying with the Gaussian distribution, are generated to emulate the imperfect
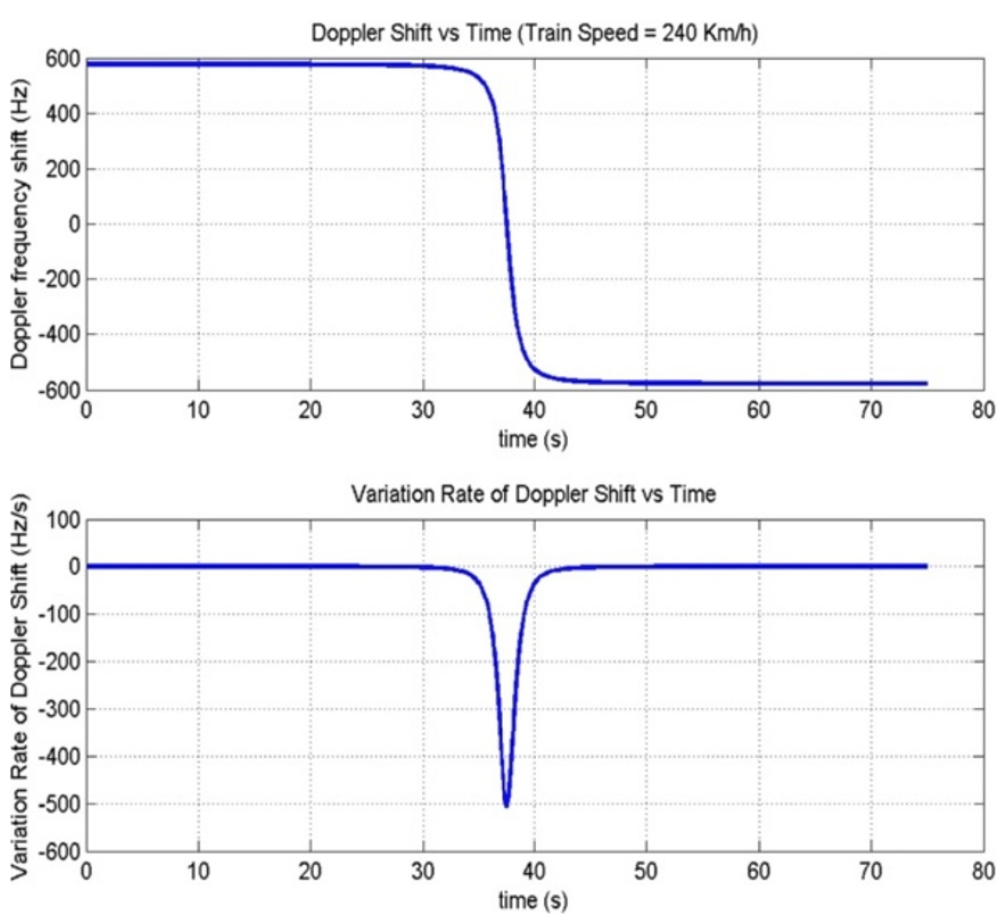

Figure 10 Doppler frequency shift versus time and Doppler variation rate versus time for the LOS path signal. 


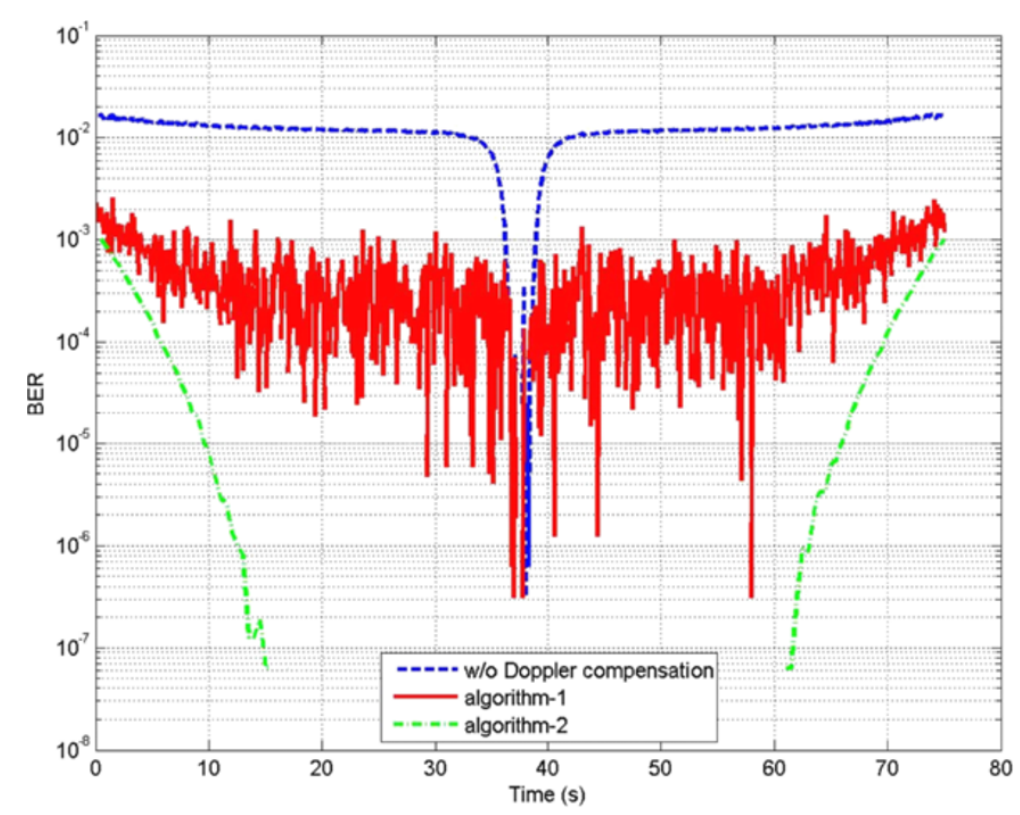

Figure 11 BER versus time under LOS scenarios.

channel parameters in the REM. Furthermore, considering that the estimation theory, the variance of channel estimation error is a function of the channel conditions, especially, the SNR. In general, the higher the SNR, the smaller the variance of the channel estimation errors. Therefore, in the simulation corresponding to Figure 13, the error of channel coefficient and angle of arrival are assumed to comply with the following zero-mean normal distributions (refer to Chapter 3 in [34]), respectively:
The estimation error of the angle of arrival $(\Delta \theta)$ and the fading coefficient of the received signal $(\Delta \alpha)$ are random variables following zero-mean Gaussian distributions:

$$
\Delta \theta \sim N\left(0,\left(\frac{1}{\pi \sqrt{\gamma}}\right)^{2}\right)
$$

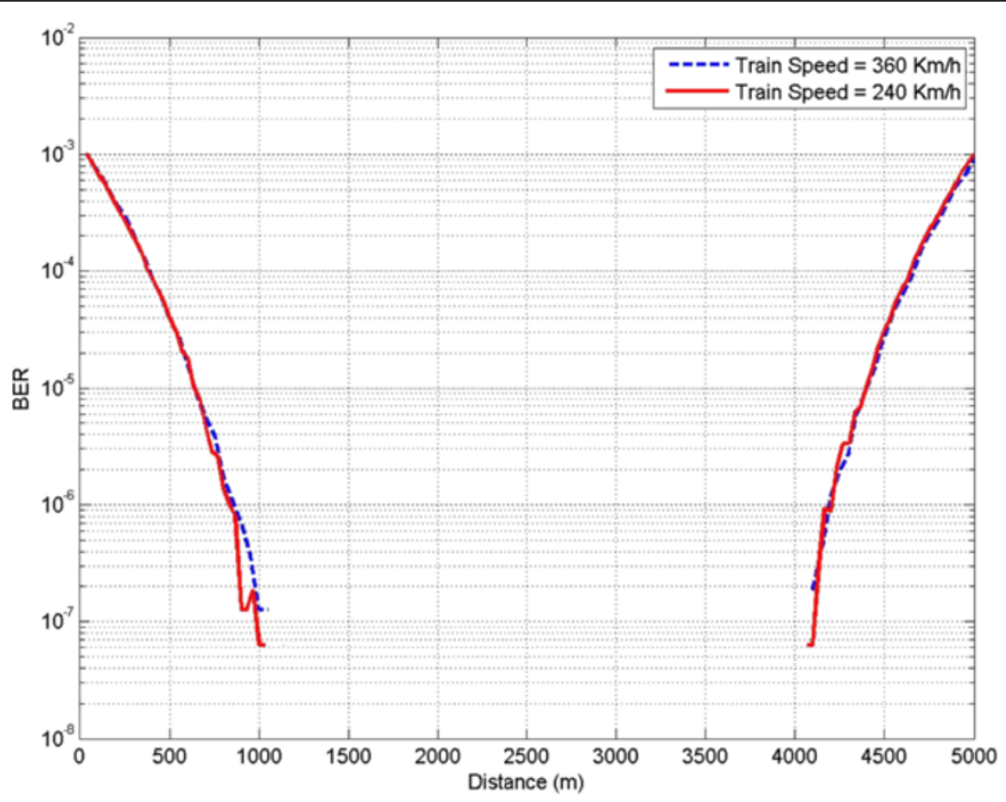

Figure 12 BER versus distance at different train speeds (assuming perfect channel information is employed in the advanced cognitive Doppler compensation algorithm). 


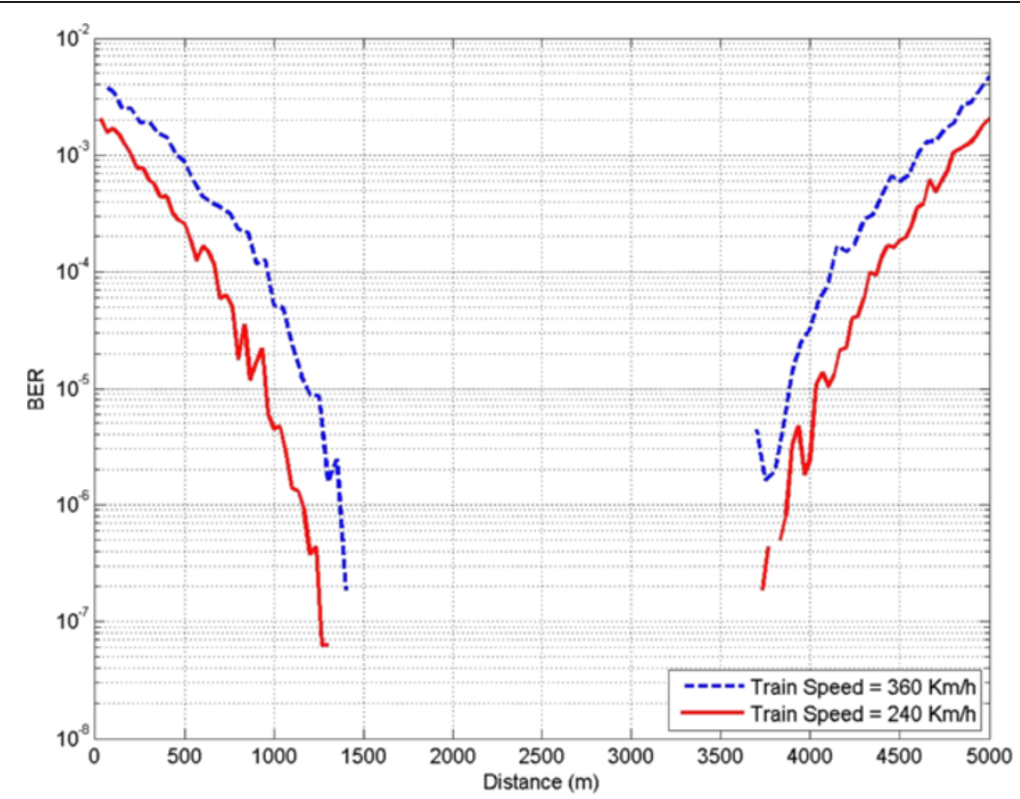

Figure 13 Impact of imperfect channel information on the BER performance (two different train speeds-240 and $360 \mathrm{~km} / \mathrm{h}$ are simulated).

$$
\Delta \alpha \sim\left(0, \frac{1}{\gamma}\right)
$$

where $\gamma$ is the SNR.

Another important factor that may degrade the performance of the REM-based cognitive Doppler spread compensation algorithms is the positioning error.
Positioning errors are inevitable in practice even though the accuracy of train positioning could be on the order of $10^{-2} \mathrm{~m}$ when employing the state-of-the-art positioning technologies (as detailed in Section "Positioning accuracy of the high-speed train"). The impact of train positioning error on link BER performance is also simulated in this article. The spatial sampling distance $\left(L_{\mathrm{s}}\right)$ in the REM for high-speed rail should be no greater than the channel coherence distance $\left(L_{\mathrm{c}}\right)$. Note that $L_{\mathrm{c}}$ is

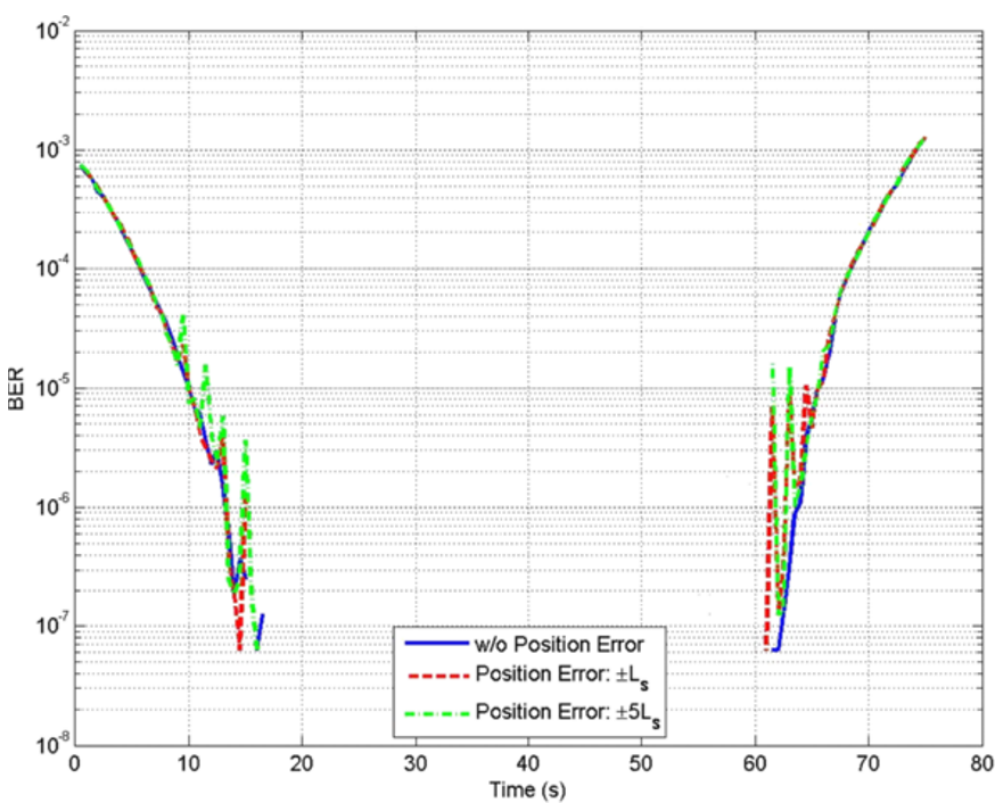

Figure 14 Impact of train positioning errors on the BER performance (train speed $=240 \mathrm{~km} / \mathrm{h}$ ). 


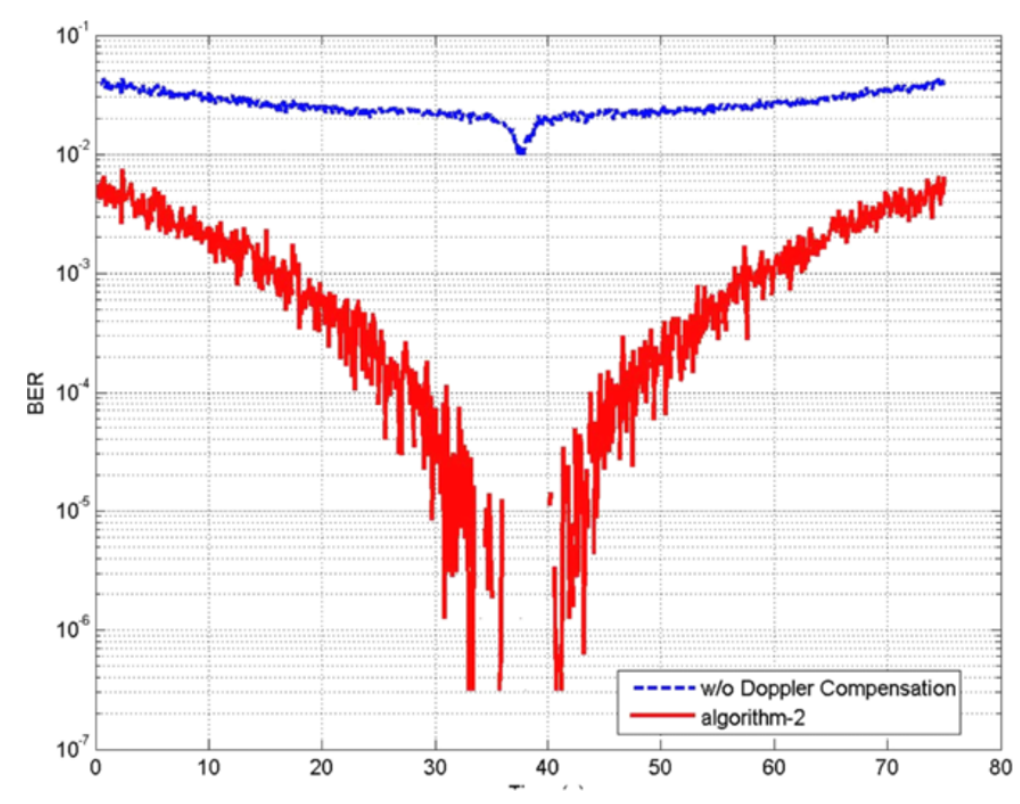

Figure 15 BER versus time under NLOS Rayleigh fading scenarios.

determined by the product of channel coherent time and the train speed. Approximately, $L_{\mathrm{c}}$ equals to half the wavelength of the central carrier frequency. Therefore, the higher the carrier frequency, the shorter the channel coherence distance. In this simulation, carrier frequency is $2.6 \mathrm{GHz}, L_{\mathrm{c}} \approx 5.8 \mathrm{~cm} . L_{\mathrm{s}}$ is set as $3 \mathrm{~cm}$; the train speed is $240 \mathrm{~km} / \mathrm{h}$; the train positioning error is assumed to be $\pm L_{\mathrm{s}}$ and $\pm 5 L_{\mathrm{s}}$, respectively. The simulated BER performance (as shown in Figure 14) indicates that when the positioning errors are considered, the link BER performance degrades to some extent during a small portion of the whole simulation period. Interestingly note that the BER degradation due to the positioning error is almost negligible, during some portion of the simulation period (i.e., from 0 to $9 \mathrm{~s}$ and from 66 to $75 \mathrm{~s}$, when the train is far away from the track-side base station and the corresponding SNR is relatively low, and from 16 to $61 \mathrm{~s}$, when the train gets closer to the BS and the SNR is fairly high); during the transition periods (i.e., from 9 to $16 \mathrm{~s}$ and from 61 to $66 \mathrm{~s}$ ), the degradation of radio link BER performance is most

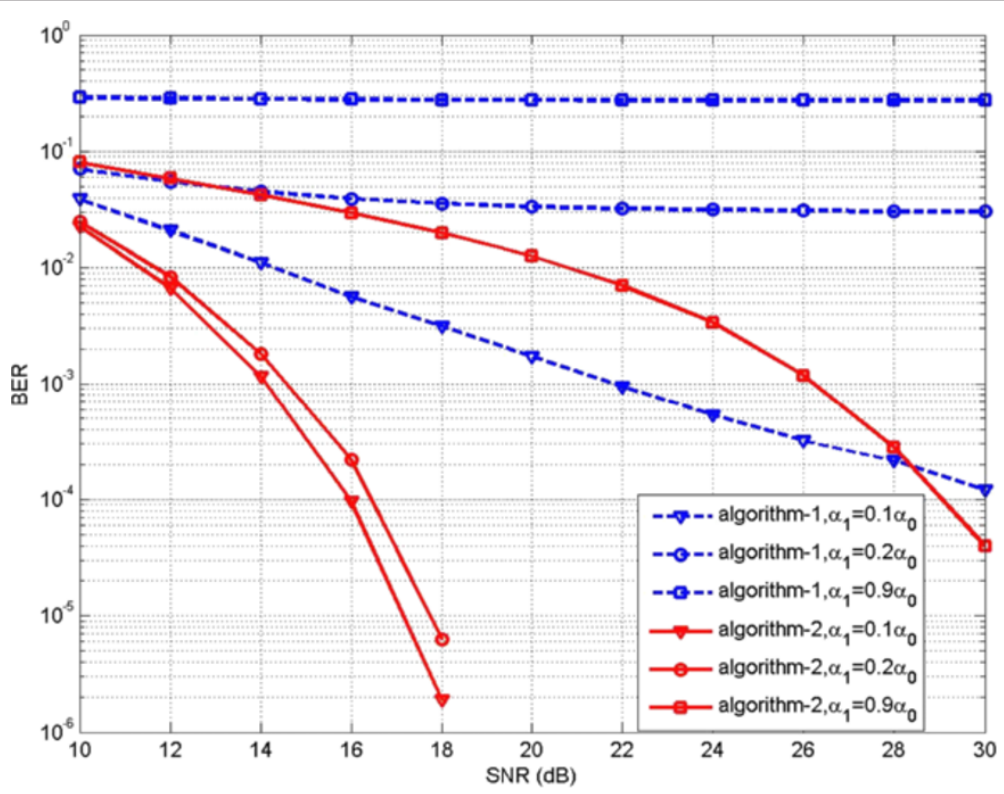

Figure 16 BER versus SNR when using two different Doppler spread compensation algorithms. 

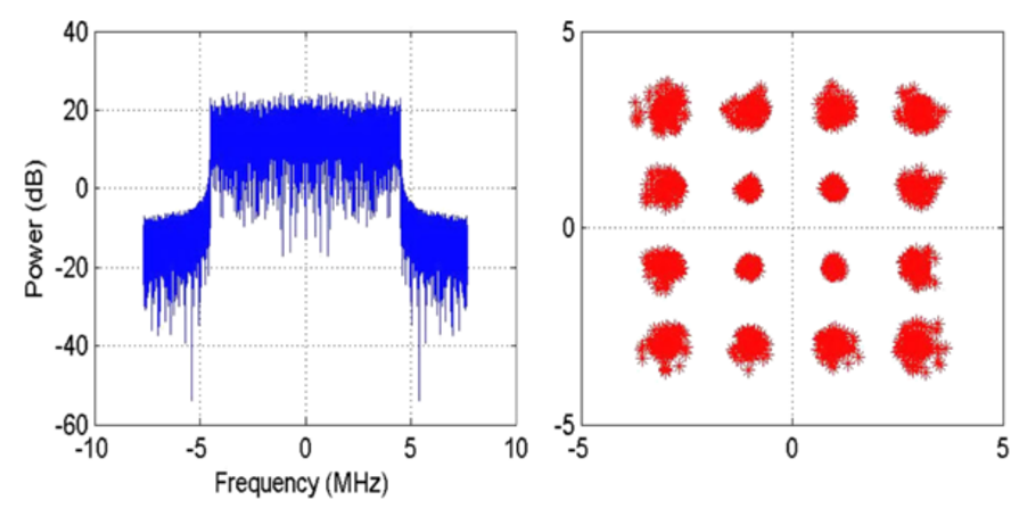

Figure 17 Received signal spectrum (left) and constellation of the demodulated signal (right), (the simple Doppler compensation algorithm is employed here).

pronounced. In addition, as can be seen from Figure 14, the larger the train positioning error, the more severe degradation it may result in.

\section{Simulation results and analysis for NLOS scenarios}

As shown in Figure 15, under the NLOS scenarios (train speed of $240 \mathrm{~km} / \mathrm{h}$ ), by employing the advanced Doppler compensation algorithm based on the dedicated REM for high-speed rail (marked as Algorithm 2 in this figure), the cognitive broadband mobile communication system has significant performance gain against the approach without Doppler compensation.

From the simulations as shown in Figure 15, we observed that, without channel coding and interleaving, under the NLOS scenarios, the BER performance of TD-LTE-based high-speed rail broadband mobile communication system is worse than the BER performance under LOS scenarios when using the same REM-based Doppler compensation algorithm. To understand this phenomenon, the BER versus SNR is investigated and plotted in Figure 16 under different channel conditions.
In Figure 16, it is assumed that the main path has a Doppler frequency shift of $577 \mathrm{~Hz}$, whereas the second path has a Doppler shift of $-577 \mathrm{~Hz}$ (which is the worst-case scenario for Doppler compensation in our simulations as the Doppler spread is maximized with such assumption). Figure 16 shows that the advanced Doppler compensation algorithm (marked as Algorithm 2 in this figure) can achieve significantly better performance than the simple Doppler compensation algorithm (marked as Algorithm 1 in this figure). Figure 16 also indicates that, even under the serious Doppler spread scenario (corresponding to the case of $\left.\alpha_{1}=0.9 \alpha_{0}\right)$, the advanced Doppler compensation algorithm can still work (no BER error floor), whereas there is an obvious BER error floor resulting from a mild Doppler spread (corresponding to the case of $\alpha_{1}=0.2 \alpha_{0}$ ) if the simple Doppler compensation algorithm is employed.

The received signal spectrum and constellation of the demodulated signal after Doppler compensation are also plotted in Figures 17 and 18 with regarding to two different second path power levels: $20 \mathrm{~dB}$ or $14 \mathrm{~dB}$ weaker than the main path power level, respectively. It shows from Figures 16, 17, and 18 that when the Doppler
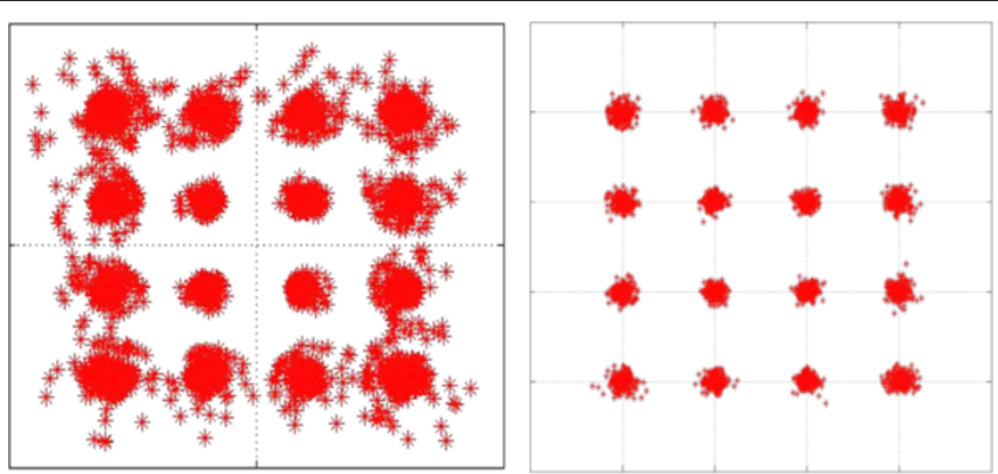

Figure 18 Constellation of the demodulated signal (left: using the simple Doppler compensation algorithm; right: using the advanced Doppler compensation algorithm). 
spread gets larger (as the case when the second path is $14 \mathrm{~dB}$ weaker than the main path, i.e., $\left.\alpha_{1}=0.2 \alpha_{0}\right)$, the performance of simple Doppler compensation algorithm gets worse, resulting in poorer OFDM demodulation and higher BER, for example, the BER at $30 \mathrm{~dB}$ SNR raises from $10^{-4}$ to $3 \times 10^{-2}$ when using the simple Doppler compensation algorithm, as shown in Figure 16. The effectiveness of the advanced Doppler compensation algorithm against the simple Doppler compensation algorithm is also clearly demonstrated from the constellation diagrams of the demodulated signal: as shown in Figure 18, when using the simple Doppler compensation algorithm, the demodulated OFDM symbols are scattered very widely, which results in higher BER.

\section{Conclusion}

Broadband mobile communications are critical to the quality of passenger experience, safety, and operational efficiency for high-speed trains. The demand on highly reliable broadband mobile communications for high-speed rail is ever increasing as more and more high-speed railways are under construction around the world.

Considering the unique features of high-speed rail broadband mobile communications, especially the repetitive movement of high-speed train along a fixed course, this article presents novel Doppler spread compensation algorithms based on the dedicated REM for railway. REMbased Doppler spread compensation algorithms have significant advantages, e.g., it can reduce the processing load resulting from repetitive and complicated channel estimations along the high-speed rail. Link-level performances of an OFDM-based high-speed rail broadband mobile communication system are evaluated under dynamically changing radio channels (including both LOS and NLOS scenarios), emulating the movement of high-speed train along a given course. Both analytical analysis and simulation results show that the proposed REM-based Doppler spread compensation algorithm can significantly improve the link performance of OFDM-based broadband mobile communication systems under various high-speed rail scenarios. The impact of imperfect channel information and train positioning errors on the link BER performance has also been simulated and analyzed in this article. For the future work, the performance of REM-based cognitive Doppler spread compensation algorithms need to be further validated with simulations and/or field test, taking other practical issues (such as the carrier frequency offset and phase noise of the local oscillators) into account.

\section{Competing interests}

The authors declare that they have no competing interests.

\section{Acknowledgment}

This study was supported in part by the NSFC project under grants Nos. 61132003 and 61032002, and Beijing Jiaotong University (2011JBM013 and 2011R(004).
Received: 15 February 2012 Accepted: 26 July 2012

Published: 17 August 2012

\section{References}

1. Wikipedia, High-speed rail, http://en.wikipedia.org/wiki/High-speed_rail

2. Y Zhao, J Li, Analysis of the impact of Doppler spread on OFDM-based nextgeneration high-speed rail broadband mobile communications. in Proceedings of IET International Conference on Communication Technology and Application (Beijing, China, 2011), pp. 116-120

3. K Yamada, Y Sakai, T Suzuki, Y Kawahara, T Asami, H Aida, A communication system with a fast handover under a high speed mobile environment. in Proceedings of IEEE Vehicular Technology Conference (Ottawa, Canada, 2010), pp. 1-5

4. T Gao, B Sun, A high-speed railway mobile communication system based on LTE, in Proceedings of 2010 International Conference on Electronics and Information Engineering, vol. 1st edn. (Kyoto, Japan, 2010), pp. 414-417

5. UIC Project EIRENE System Requirements Specification 15.0,17

6. C Tao, L Liu, J Qiu, Z Tan, System architecture and key technologies for high-speed rail broadband wireless access. Telecom. Sci. (in Chinese) 26(6), 95-101 (2010)

7. MJF-G Garcia, O Edfors, JM Paez-Borrallo, Frequency offset correction for coherent OFDM in wireless systems. IEEE Trans. Consum. Electron 47(1), 187-193 (2001)

8. M Luise, M Marselli, R Reggiannini, Low-complexity blind carrier frequency recovery for OFDM signals over frequency-selective radio channels. IEEE Trans. Commun. 50(7), 1182-1188 (2002)

9. C Muschallik, Improving an OFDM reception using an adaptive Nyquist windowing. IEEE Trans. Consum. Electron. 42(3), 259-269 (1996)

10. E Peiker, WG Teich, J Lindner, Windowing in the receiver for OFDM systems in high-mobility scenarios. Multi-Carrier Syst. Solutions 2009(41), 57-65 (2009)

11. Y Zhao, SG Haggman, Intercarrier interference self-cancellation scheme for OFDM mobile communication systems. IEEE Trans. Commun 49, 1185-1191 (2001)

12. A Seyedi, GJ Saulnier, General ICl self-cancellation scheme for OFDM systems. IEEE Trans. Veh. Technol. 54(1), 198-210 (2005)

13. G Renhua, S Shanlin, ICl performance analysis for all phase OFDM systems. J. Electromag. Anal. Appl. 1(2), 118-123 (2009)

14. P Klenner, KD Kammeyer, Doppler-compensation for OFDM transmission by sectorized antenna reception. Eur. Trans. Telecomms. 19(5), 571-579 (2008)

15. L Liu, C Tao, J Qiu, H Chen, A performance study for sectorised antenna based doppler diversity in high-speed railway communications. Radioengineering 20(4), 866-871 (2011)

16. $Y$ Zhao, $B$ Le, JH Reed, in Network support: the radio environment map, ed. by $F$ Bruce. Cognitive Radio Technology (Elsevier, Amsterdam, 2006), pp. 337-363

17. Y Zhao, S Mao, J Neel, JH Reed, Performance evaluation of cognitive radios: metrics, utility functions and methodologies. Proc. IEEE (Special Issue on Cognitive Radio) 97(4), 642-659 (2009)

18. Y Zhao, $S$ Mao, JH Reed, Y Huang, Utility function selection for streaming videos with a cognitive engine testbed. ACM/Springer Mob. Netw. Appl. (MONET) (Special Issue on Advances in Wireless Testbeds and Research Infrastructures) 15(3), 446-460 (2010)

19. J Mitola, GQ Jr Maguire, Cognitive radio: making software radios more personal. IEEE Personal Commun. 6(4), 13-18 (1999)

20. S Haykin, Cognitive radio: brain-empowered wireless communications. IEEE J. Sel. Areas Commun. 23(2), 201-220 (2005)

21. IF Akyldiz, W-Y Lee, MC Vuran, S Mohanty, Next generation dynamic spectrum access cognitive radio wireless networks: a survey. Int. J. Comput. Telecommun. Netw. 50(13), 2127-2159 (2006)

22. BA Fette (ed), Cognitive Radio Technology (Elsevier, Amsterdam, 2006)

23. K Byung-Chul, L I-Tai, Doppler diversity for OFDM wireless mobile communications. Part l: frequency domain approaches, in Proceedings of IEEE Vehicular Technology Conference, vol. 4th edn. (VTC 2003-Spring, Orlando, FL, 2003), pp. 2677-2681

24. AM Sayeed, B Aazhang, Joint multipath-Doppler diversity in mobile wireless communications. IEEE Trans. Commun 47, 123-132 (1999)

25. X Wang, G Zhu, X Chen, Z Tan, Doppler diversity for OFDM high-speed mobile communications, in Proceedings of IEEE International Conference on Communications, vol. 10th edn. ( Istanbul, Turkey, 2006), pp. 4665-4669

26. C Tao, J Qiu, L Liu, A novel OFDM channel estimation algorithm with ICl mitigation over fast fading channels. Radioengineering 19(2), 347-355 (2010) 
27. L Liu, C Tao, J Qiu, A novel comb-pilot transform domain frequency diversity channel estimation for OFDM systems under doubly fading channels. J. China Railway Sci. 32(2), 119-124 (2010)

28. L Xiong, X Lu, Z Zhong, B Ai, M Zhu, Simulation on the characteristics of the wireless channel for high-speed railway GSM-R system. J. China Railway Sci. 31(5), 84-89 (2010)

29. Y Zhao, C Yin, J Li, Learning and optimization-based channel estimation for cognitive high-speed rail broadband wireless communications, in Proceedings of 2012 International Symposium on Communications and Information Technologies, (Gold Coast, Australia, 2012), pp. 1-6

30. B Cai, J Wang, Q Yin, I Liu, A GNSS based slide and slip detection method for train positioning, in Proceedings of 2009 Asia-Pacific Conference on Information Processing (Shenzhen, China, 2009), pp. 450-453

31. Y Zhang, D Zhou, H Liu, B Liu, Research on multi-sensor information fusion based CBTC train speed and position measurement. Urban Rapid Rail Transit 24(4), 30-32 (2011)

32. C Qian, D Shao, W Xie, Z Han, Design and research on speed and position detection system for high-speed Maglev train. Instrum. Tech. Sensor 9 , 34-36 (2004)

33. Y Han, EP Stuntebeck, JT Stasko, GD Abowd, A visual analytics system for radio frequency fingerprinting-based localization, in Proceedings of 2009 IEEE Symposium on Visual Analytics Science and Technology (Atlantic City, USA 2009), pp. 35-42

34. SM Kay, Fundamentals of Statistical Signal Processing, Vol. I: Estimation Theory (Pearson Education, New Jersey, 1993)

35. P Kyösti, J Meinilä, L Hentilä, X Zhao, T Jämsä, C Schneider, M Narandzić, M Milojević, A Hong, J Ylitalo, V-M Holappa, M Alatossava, R Bultitude, Y Jong, T Rautiainen, WINNER I| D1.1.2, WINNER I/ channel models, Online Available https:// www.ist-winner.org/deliverables.html, September 2007

36. TS Rappaport, Wireless Communications: Principles and Practice (Prentice Hall (Englewood Cliffs, NJ, 1996)

37. WC Jakes (ed), Microwave Mobile Communications (IEEE Press, New York, 1994)

doi:10.1186/1687-1499-2012-263

Cite this article as: $L i$ and Zhao: Radio environment map-based cognitive Doppler spread compensation algorithms for high-speed rail broadband mobile communications. EURASIP Journal on Wireless Communications and Networking 2012 2012:263.

\section{Submit your manuscript to a SpringerOpen ${ }^{\circ}$ journal and benefit from:}

- Convenient online submission

- Rigorous peer review

- Immediate publication on acceptance

- Open access: articles freely available online

- High visibility within the field

- Retaining the copyright to your article

Submit your next manuscript at $\boldsymbol{\wedge}$ springeropen.com 